\title{
Evidências de validade do Roteiro de Avaliação da Consciência Fonológica (RACF)
}

\section{Validity evidence for the Protocol for Phonological Awareness Task (PPAT)}

\section{Evidencias de validad de la hoja de ruta de evaluación de la conciencia fonológica (RACF)}

\section{Márcia Maria Peruzzi Elia da Mota*}

Universidade do Estado do Rio de Janeiro - UERJ, Rio de Janeiro, Rio de Janeiro, Brasil

\author{
Acácia Aparecida Angeli dos Santos** \\ Universidade São Francisco - USF, Itatiba, São Paulo, Brasil
}

\section{Silvia Brilhante Guimarães***}

Universidade do Estado do Rio de Janeiro - UERJ, Rio de Janeiro, Rio de Janeiro, Brasil

\section{Carolina Conti $* * * *$}

Universidade Federal de Juiz de Fora - UFJF, Juiz de Fora, Minas Gerais, Brasil

\begin{abstract}
RESUMO
O presente estudo investigou a validade do instrumento Roteiro de Avaliação da Consciência Fonológica - RACF. Participaram da pesquisa 217 crianças de escolas públicas matriculadas no $2^{\circ}, 3^{\circ}$ e $4^{\circ}$ ano do ensino fundamental. As crianças foram entrevistadas em quatro sessões nas quais procedeu a aplicação dos instrumentos: RACF, subteste de leitura do TDE e subtestes de vocabulário e de dígito do WISC III. Foram encontradas evidências de validade de critério, pois os escores se diferenciaram pelos anos escolares e nível de habilidade em leitura. Outra evidência de validade foi detectada quando se verificou a associação dos escores do RACF com outras tarefas de avaliação cognitiva, a saber, o vocabulário e memória para dígitos. Enfim, os resultados mostram que o RACF é uma tarefa sensível para mensurar a habilidade metafonológica das crianças.

Palavras-chaves: validade de critério, consciência metalinguística, habilidades linguísticas, avaliação psicológica.
\end{abstract}

\section{ABSTRACT}

The present study investigated the validity of the instrument Protocol for Phonological Awareness assessment". Two-hundred and seventeen children drawn from public schools enrolled in the 2nd, 3rd and 4th year of elementary school participated in the research. The children were interviewed in four sessions in which the application of the following 
instruments was carried out in four sessions: RACF, TDE: reading subtest and the WISC III: vocabulary and digit subtest. Evidence of validity for discrimination between series was found. The results indicated that phonological awareness develops throughout the first years of schooling. Evidence of validity for RACF was also met when its association with other tasks such as vocabulary and working memory was found. In summary, our results show that the RACF is a task that helps you access the metaphonological ability of children.

Keywords: criterion validity, metalinguistic awareness, linguistic abilities.

\begin{abstract}
RESUMEN
Este estudio investigó La validez del instrumento Roteiro de Avaliação da Consciência Fonológica - RACF.Participaron de la investigación 217 niños del $2^{\circ}$ a $4^{\circ}$ grado de escuelas públicas de la enseñanza primaria. Los niños fueron entrevistados en cuatro sesiones en las cuales se aplicaron dos instrumentos: RACF, subtest de lectura del TDE y subtestes de vocabulario y de dígitos del WISC III. Fueron encontradas evidencias de validez de criterio, una vez que las puntuaciones se diferenciaron por los grados escolares y el nivel de habilidad en lectura. Otra evidencia de validez fue detectada cuandose verificó la asociación de las puntuaciones del RACF con otras tareas de evaluación cognitiva, a saber, el vocabulario y la memoria para dígitos. De esa forma, los resultados muestran que el RACF es una tarea sensible para medir la habilidad fonemica de los niños.
\end{abstract}

Palabras clave: validez de critério, conciencia metalinguística, habilidades lingüísticas.

\title{
1 Introdução
}

A literatura na área PSI tem destacado o papel do processamento fonológico no desempenho da aquisição da escrita. Três habilidades fonológicas parecem estar envolvidas no desenvolvimento da leitura de palavras através da rota alfabética: a consciência fonológica, a memória auditiva de trabalho, e a recuperação dos códigos fonológicos que correspondem às letras ou grupos de letras na grafia das palavras (Wagner \& Torgesen, 1987; Castles \& Coltheart, 2004, Spinillo, Mota, \& Correa, 2010, entre outros). Neste trabalho focaremos consciência fonológica.

Estudos em diversas ortografias alfabéticas têm confirmado resultados que enfatizam o papel da consciência fonológica na alfabetização (Alloway et al., 2005; Bradely \& Bryant, 1985; Cuninghan, 1990; Goswami \& Bryant, 1990). No Português do Brasil, os trabalhos de Barrera e Maluf (2003), Capovilla e Capovilla (2000), Guimarães (2003), Miranda e Mota, 2011; Pestun, Omote, Barreto e Matsuo (2010), Golbert e Salles (2010), Santos (1996), Souza e Bandini (2007), Suehiro e Santos (2011), só para citar alguns, ressaltam a importância do tema para aquisição da língua escrita. Consciência fonológica pode ser definida como a habilidade de refletir sobre os sons que compõem a fala (Cardoso-Martins, 1995) e é parte de uma habilidade mais geral chamada consciência metalinguística 
(Gombert, 1992). Consciência metalinguística envolve a reflexão da linguagem como objeto do pensamento e difere da percepção e da produção da fala. Enquanto as últimas envolvem um conhecimento tácito da linguagem, a primeira envolve a manipulação intencional das unidades linguísticas (Gombert, 2003).

Não há uma forma única de se avaliar a consciência fonológica. Diferentes unidades linguísticas podem fazer parte da consciência fonológica, havendo diferentes graus de manipulação envolvidos na avaliação dessas unidades. Há tarefas que envolvem a habilidade de manipular explicitamente os segmentos fonêmicos da fala, outras que envolvem a detecção de rima e aliteração (CardosoMartins, 1995; Cardoso-Martins \& Frith, 1999). As autoras observam que ao contrário das habilidades de manipular explicitamente os seguimentos fonêmicos da fala, a de detectar rima e aliteração parece estar mais associada a um julgamento global da semelhança fonológica entre as palavras.

Outros estudos na área mostram que essas tarefas são mais simples do que as tarefas que exigem manipulação e/ou segmentação explícita de unidades fonêmicas. De fato, estudos estrangeiros e nacionais têm demonstrado que já na educação infantil crianças realizam tarefas de detecção de rima e aliteração com sucesso (Bradley \& Bryant, 1983; MacLean, Bryant, \& Bradley, 1987; Phillips, Clancy-Manchetti \& Lonigan, 2008; Pestun, Omote, Barreto, \& Matsuo, 2010; Suehiro \& Santos, 2011).

No estudo clássico de Bradley e Bryant (1983), os autores pediram a crianças na educação infantil que julgassem três palavras (por exemplo, "gato", "mato" e "toca"). As crianças tinham que decidir qual das palavras não continha o mesmo som das outras duas. Os sons investigados envolviam a rima ou a aliteração. Essa tarefa ficou conhecida como The Oddity Test e tem sido amplamente utilizada desde a publicação desse estudo.

Os resultados do estudo de Bradley e Bryant (1983) mostraram que as crianças com melhor desempenho no Oddity Test eram também as crianças com melhor desempenho na leitura quando foram alfabetizadas. Pode-se dizer também que esse estudo despertou 0 interesse dos pesquisadores para importância dessa habilidade para alfabetização. Os autores defendiam a posição de que a consciência metalinguística é um precursor da alfabetização. Entretanto, pesquisas realizadas com tarefas que envolviam uma manipulação mais explícita dos fonemas, e não unidades como a rima e a aliteração apontavam para um modelo diferente do ora proposto.

Pesquisas realizadas com o propósito de desafiar o modelo de Bradley e Bryant (1983) identificaram que sujeitos não alfabetizados tinham dificuldade em manipular os fonemas, o que levou os autores a argumentarem que a alfabetização promovia a habilidade metalinguística (Bertelson, Gelder, Tfouni \& Morais, 1989; Morais, 
Content, Bertelson, Cary, \& Alegria, 1986). Anos mais tarde, o avanço no conhecimento sobre o tema tem indicado que alguma habilidade metalinguística é necessária para alfabetização, mas que as habilidades verdadeiramente metalinguísticas dependem da experiência com a língua escrita (Gindri, Keske-Soare, \& Mota, 2007; Gombert, 2003; Mota \& Guimarães, 2011).

Gombert (1992) chama atenção para a importância da escolha da tarefa para avaliar a consciência metalinguística. Tarefas que não envolvem a manipulação direta e intencionais dos sons da fala não podem ser consideradas 'metalinguísticas', pois envolveriam aspectos perceptivos mais globais. Estudos também têm demonstrado que não é apenas a unidade linguística avaliada que afeta o desempenho na tarefa, visto que a posição dos sons na palavra também interfere no desempenho (Cardoso-Martins, 1995).

Tem havido um aumento da preocupação em se estabelecer a validação de tarefas que avaliam a consciência fonológica. Assim estudiosos estrangeiros (Branum-Martin et al., 2006) e brasileiros (Santos, 1996, Suehiro \& Santos, 2011) têm se dedicado à pesquisa das qualidades psicométricas de instrumentos com o propósito de verificar se eles medem aquilo a que se propõem a medir conforme preconizam os psicometristas (Urbina, 2007; Nunes \& Primi, 2010).

Se algum grau de habilidade metalinguística é necessário para a aquisição da escrita, tarefas que avaliam essa habilidade são de grande importância educacional e clínica. Foi instigado por essa questão de pesquisa que o presente estudo foi delineado, pressupondo que esta é uma questão relevante porque os seus resultados podem ajudar a se pensar em intervenções que favoreçam o desenvolvimento dessa habilidade, em crianças que mostrem dificuldades nessas tarefas, tal como bem exemplificado no estudo de Souza e Bandini (2007) com crianças surdas bilíngues.

Dada a importância do tema para a alfabetização e a importância da alfabetização para o desenvolvimento escolar da criança, o acesso a tarefas bem construídas com evidências de validade estabelecidas é importante para profissionais ligados à área, visto que os resultados obtidos poderão possibilitar inferências mais adequadas para sua interpretação. Sob essa perspectiva, o objetivo do presente estudo foi investigar evidências de validade de um Roteiro de Avaliação da Consciência Fonológica, proposta por Santos (1996) com base nos mesmos princípios da tarefa de Bradley e Bryant (1983). Vale destacar que serão investigadas evidências de validade de critério (ano escolar e tipo de leitor) e evidências relativas à correlação com medidas que avaliam construtos similares (processamento fonológico e desempenho em leitura).

\section{Análise dos dados}


Para testar a validade de critério do Roteiro de Avaliação da Consciência Fonológica, uma ANOVA fatorial, foi realizada com o número de respostas corretas no RACF como variável dependente e $\mathrm{o}$ ano escolar (segundo, terceiro e quarto) e habilidade na leitura (superior, média e inferior) como variáveis independentes. Por sua vez, para verificar a evidência de validade do instrumento pela sua comparação com medidas que avaliam construtos similares recorreuse à prova de correlações bisseriais de Pearson.

\section{Método}

\section{Participantes}

Participaram desse estudo uma amostra de 217 crianças, 43 de segundo ano, 85 de terceiro ano e 89 de quarto ano do ensino fundamental do projeto "Avaliação psicométrica de medidas de consciência metalinguística". As crianças eram alunos de escolas da rede pública de uma cidade no interior de Minas Gerais. O critério de inclusão no estudo foi a assinatura do Termo de Consentimento Livre Esclarecido (TCLE) por parte dos pais das crianças.

\section{Instrumentos}

Roteiro de Avaliação de Consciência Fonológica - RACF (Santos, 1996).

A tarefa consiste de três séries de itens, cada uma com cinco itens e dois exemplos, que visam avaliar a dificuldade na identificação do fonema inicial, final e o do meio das palavras. Cada item é composto de uma palavra modelo e outras três, para alternativas de resposta. Ex. de item com som inicial, som final e som medial, respectivamente.

$$
\begin{array}{lr}
\frac{\text { copo }}{\text { bondade }} & \begin{array}{l}
\text { cobra - sapo - lata } \\
\text { apagado - bandido - felicidade } \\
\text { desejar }
\end{array} \\
\text { desligar - gazeta - trabalhar }
\end{array}
$$

O teste apresenta evidência de validade preditiva demonstrada no estudo inicial da autora (Santos, 1996), bem como evidências de validade de critério, na qual foi identificada sua sensibilidade para separar os testados por série (Suehiro \& Santos, 2011). A cada acerto a criança recebe um ponto, podendo alcançar no máximo 15 pontos. 
Escala de Inteligência Wechsler para crianças-WISC III (Weschler, 1991)

Apenas foram utilizados os subtestes de Vocabulário e Dígitos (memória auditiva), recorrendo-se ao uso dos escores ponderados para as análises. Destaca-se que os dois subtestes da escala Wechsler escolhidos apresentam bons critérios de consistência interna medida pelo critério de Guttman (coeficientes maiores que $0,60)$. A utilização de tais subtestes se deu visando ao controle da influência do desenvolvimento cognitivo na aquisição da leitura e escrita. A escolha do WISC III se deveu ao fato de ser um teste padronizado para uma amostra brasileira e ser um teste amplamente utilizado na literatura nacional e internacional da área.

Teste de Desempenho Escolar - TDE (Stein, 1994).

Para esta pesquisa utilizou-se apenas o subteste de leitura. Neste a criança deve ler em voz alta um conjunto de 70 palavras isoladas do contexto distribuídas em uma única folha. A tarefa apresenta evidência de validade de critério e bom índice de consistência interna $(a>0,70)$. O subteste foi administrado e corrigido de acordo com as instruções e procedimentos especificados do manual. A cada acerto é dado um ponto e para cada erro zero ponto, perfazendo um total máximo de 70 pontos.

\section{Procedimentos}

O presente estudo faz parte de uma pesquisa mais ampla que incluiu a aplicação de outras provas de avaliação das competências cognitivas e, em especial, da leitura. Após o consentimento dos responsáveis expresso pela assinatura no Termo de Consentimento Livre e Esclarecido eram aplicados, de forma individual, nos alunos os instrumentos descritos a seguir. A aplicação foi feita durante o período letivo, na própria escola dos participantes, no turno em que eles frequentavam a escola.

As aplicadoras eram pessoas com experiência na área e integrantes do projeto mencionado.

$\mathrm{Na}$ aplicação do Roteiro de Avaliação da Consciência Fonológica (RACF), na primeira série de palavras, o aplicador fala a palavramodelo e informa à criança que serão ditas outras três palavras e que ela deverá indicar entre elas qual a que começa com o mesmo som. 0 mesmo foi feito com as duas outras séries que focalizam o som final e depois o som do meio. As duas primeiras palavras-modelo de cada série são utilizadas como exemplo, não tendo sua pontuação computada. A aplicação e a correção dos subtestes do WISC III e do subteste de leitura do TDE corresponderam às instruções expressas 
no manual de aplicação de cada teste padronizado.

\section{Resultados}

Os resultados foram analisados em razão dos objetivos propostos. Dessa forma, serão relatados respeitando-se a sequência em que foram estabelecidos.

Com vistas ao estabelecimento da evidência de validade de critério relativa ao ano escolar, iniciou-se a comparação entre os escores obtidos em cada um deles. Para tanto, recorreu-se à análise de variância. Aplicou-se um teste post-hoc sempre que diferenças estatisticamente significativas foram encontradas na ANOVA. A Tabela 1 mostra os resultados alcançados.

\begin{tabular}{|c|c|c|c|c|}
\hline & Roteiro & onscié & ologica. & \\
\hline Ano & $\begin{array}{c}\text { Habilidades de } \\
\text { leitura }\end{array}$ & $N$ & Média & Desvio Padrão \\
\hline $2^{\circ}$ & Superior & 14 & 11,43 & 2,8 \\
\hline & Média & 27 & 11,07 & 2,15 \\
\hline & Inferior & 2 & 10,50 & 0,71 \\
\hline & Total & 43 & 11,16 & 2,31 \\
\hline $3^{\circ}$ & Superior & 24 & 13,00 & 1,96 \\
\hline & Média & 45 & 11,04 & 2,50 \\
\hline & Inferior & 16 & 9,63 & 2,16 \\
\hline & Total & 85 & 11,33 & 2,56 \\
\hline $4^{\circ}$ & Superior & 12 & 13,42 & 1,73 \\
\hline & Média & 28 & 12,96 & 2,28 \\
\hline & Inferior & 49 & 11,57 & 1,7 \\
\hline & Total & 89 & 12,26 & 2,07 \\
\hline Total & Superior & 50 & 12,66 & 2,27 \\
\hline & Média & 100 & 11,59 & 2,48 \\
\hline & Inferior & 67 & 11,07 & 2,01 \\
\hline & Total & 217 & 11,68 & 2,36 \\
\hline
\end{tabular}

Os resultados da ANOVA indicaram diferenças significativas relativas ao ano escolar $\left(F_{(2,216)}=8,47, p<0,001\right)$. Na Tabela 1 , verificase que houve um aumento gradual no desempenho no RACF acompanhando o avanço da escolaridade. Análises post hoc Student Newman Keusl mostraram um aumento significativo entre as médias do segundo e do terceiro ano quando comparadas às do quarto ano. No entanto, não houve diferenças entre os grupos de segundo e 
terceiro ano.

Para a comparação dos escores de leitores com bom desempenho e mau desempenho recorreu-se ao subteste de leitura do Teste de Desempenho Escolar (TDE), que possibilita o agrupamento de leitores em quatro categorias (superior, médio superior, médio inferior e inferior) para 020 ano e em três categorias (superior, médio e inferior) para $3^{\circ}$ e $4^{0}$ anos do ensino fundamental. Para podermos comparar os grupos de diferentes anos escolares, os escores definidos como habilidades 'média inferior' e 'média superior' do $2^{\circ}$ ano foram recodificados numa única categoria denominada como 'médio'.

A comparação entre leitores de diferentes níveis de habilidades, tal como avaliada pelo subteste de leitura do TDE, também apresentou diferença significativa $\left(F_{(2,216)}=5,79, p<0,001\right)$. Comparações post hoc Student Newman Keusl mostraram que os leitores no nível inferior e médio não diferiram em relação aos escores do RACF, porém os escores dos integrantes do grupo inferior foram significativamente mais baixos do que o dos leitores categorizados no nível superior. Não houve diferenças entre os leitores de nível médio e superior. A interação ano/nível de habilidade não foi significativa $\left(F_{(4,216)}=6,08, p=0,27\right)$. Em decorrência dos achados, nenhuma análise post hoc foi efetuada.

Foram realizadas ANOVAS fatoriais com os escores do RACF em cada unidade linguística de análise: 'som final', 'som medial' e 'som inicial' foram considerados como variável dependente. Por sua vez, o ano escolar (segundo, terceiro e quarto) e o nível de habilidade na leitura (superior, média e inferior) foram selecionados como variáveis independentes. A Tabela 2 mostra a média e o desvio padrão para cada posição do som. 
Tabela 2.

Médias e desvios-padrão dos escores de cada unidade lingulstica do

RAFC (som inicial, final e medial) por ano escolar e nivel de habilidade na leitura.

\begin{tabular}{|c|c|c|c|c|c|c|c|c|c|c|c|c|c|}
\hline \multicolumn{14}{|c|}{ Niveis de habilidades de leitura } \\
\hline Ano & & \multicolumn{3}{|c|}{ Inferior } & \multicolumn{3}{|c|}{ Médio } & \multicolumn{3}{|c|}{ Superior } & \multicolumn{3}{|c|}{ Total } \\
\hline & & $N$ & M & $D P$ & $N$ & M & $D P$ & $N$ & M & $D P$ & $N$ & $M$ & $D P$ \\
\hline & $\begin{array}{l}\text { RAC } \\
F_{1}\end{array}$ & 2 & 5 & 0 & 27 & $\begin{array}{l}4,5 \\
5\end{array}$ & $\begin{array}{l}0,6 \\
4\end{array}$ & $\begin{array}{l}1 \\
4\end{array}$ & & $\begin{array}{l}0,6 \\
5\end{array}$ & 43 & $\begin{array}{l}4, \\
6\end{array}$ & $\begin{array}{l}0,6 \\
3\end{array}$ \\
\hline & $\begin{array}{l}\text { RAC } \\
\text { F } 2\end{array}$ & 2 & 2 & 0 & 27 & $\begin{array}{l}3,1 \\
1\end{array}$ & $\begin{array}{l}1,3 \\
4\end{array}$ & $\begin{array}{l}1 \\
4\end{array}$ & $\begin{array}{l}3, \\
2\end{array}$ & $\begin{array}{l}1,8 \\
9\end{array}$ & 43 & $\begin{array}{l}3, \\
1\end{array}$ & $\begin{array}{l}1,5 \\
1\end{array}$ \\
\hline & $\begin{array}{l}\text { RAC } \\
\text { F } 3\end{array}$ & 2 & 3,5 & $\begin{array}{l}0, \\
7\end{array}$ & 27 & $\begin{array}{l}3,4 \\
1\end{array}$ & $\begin{array}{l}1,1 \\
5\end{array}$ & $\begin{array}{l}1 \\
4\end{array}$ & $\begin{array}{l}3, \\
6\end{array}$ & $\begin{array}{l}1,0 \\
1\end{array}$ & 43 & $\begin{array}{l}3, \\
5\end{array}$ & $\begin{array}{l}1,0 \\
8\end{array}$ \\
\hline $3^{\circ}$ & $\begin{array}{l}\text { RAC } \\
F_{1}\end{array}$ & 16 & $\begin{array}{l}4,5 \\
6\end{array}$ & $\begin{array}{l}0, \\
6\end{array}$ & 45 & $\begin{array}{l}4,5 \\
3\end{array}$ & $\begin{array}{l}0,6 \\
7\end{array}$ & $\begin{array}{l}2 \\
4\end{array}$ & $\begin{array}{l}4, \\
9\end{array}$ & $\begin{array}{l}0,2 \\
8\end{array}$ & 85 & $\begin{array}{l}4, \\
6\end{array}$ & 0,6 \\
\hline & $\begin{array}{l}\text { RAC } \\
\text { F } 2\end{array}$ & 16 & $\begin{array}{l}2,3 \\
1\end{array}$ & $\begin{array}{l}1, \\
1\end{array}$ & 45 & $\begin{array}{l}2,9 \\
5\end{array}$ & 1,3 & $\begin{array}{l}2 \\
4\end{array}$ & $\begin{array}{l}3, \\
8\end{array}$ & $\begin{array}{l}1,1 \\
7\end{array}$ & 85 & $\begin{array}{l}3, \\
1\end{array}$ & $\begin{array}{l}1,3 \\
2\end{array}$ \\
\hline & $\begin{array}{l}\text { RAC } \\
\text { F } 3\end{array}$ & 16 & $\begin{array}{l}2,7 \\
5\end{array}$ & $\begin{array}{l}1 \\
4\end{array}$ & 45 & $\begin{array}{l}3,5 \\
5\end{array}$ & $\begin{array}{l}1,2 \\
3\end{array}$ & $\begin{array}{l}2 \\
4\end{array}$ & $\begin{array}{l}4, \\
2\end{array}$ & $\begin{array}{l}0,9 \\
4\end{array}$ & 85 & $\begin{array}{l}3, \\
6\end{array}$ & $\begin{array}{l}1,2 \\
8\end{array}$ \\
\hline 40 & $\begin{array}{l}\text { RAC } \\
F_{1}\end{array}$ & 49 & 4,8 & $\begin{array}{l}0, \\
5\end{array}$ & 28 & $\begin{array}{l}4,7 \\
5\end{array}$ & $\begin{array}{l}0,7 \\
0\end{array}$ & $\begin{array}{l}1 \\
2\end{array}$ & 5 & 0 & 89 & $\begin{array}{l}4, \\
8\end{array}$ & $\begin{array}{l}0,5 \\
4\end{array}$ \\
\hline & $\begin{array}{l}\text { RAC } \\
\text { F } 2\end{array}$ & 49 & $\begin{array}{l}3,0 \\
6\end{array}$ & $\begin{array}{l}1, \\
3\end{array}$ & 28 & $\begin{array}{l}3,9 \\
3\end{array}$ & $\begin{array}{l}1,3 \\
5\end{array}$ & $\begin{array}{l}1 \\
2\end{array}$ & $\begin{array}{c}4, \\
1\end{array}$ & $\begin{array}{c}1,2 \\
4\end{array}$ & 89 & $\begin{array}{l}3, \\
5\end{array}$ & $\begin{array}{l}1,3 \\
8^{3}\end{array}$ \\
\hline & $\begin{array}{l}\text { RAC } \\
\text { F } 3\end{array}$ & 49 & $\begin{array}{l}3,7 \\
1\end{array}$ & $\begin{array}{l}1, \\
1\end{array}$ & 28 & $\begin{array}{l}4,2 \\
8\end{array}$ & $\begin{array}{l}0,9 \\
7\end{array}$ & $\begin{array}{l}1 \\
2\end{array}$ & $\begin{array}{l}4, \\
3\end{array}$ & $\begin{array}{c}0,6 \\
5\end{array}$ & 89 & $\begin{array}{l}3, \\
9\end{array}$ & $\begin{array}{l}1,0 \\
5\end{array}$ \\
\hline Tot & $\begin{array}{l}\text { RAC } \\
F_{1}\end{array}$ & $\begin{array}{l}0, \\
5\end{array}$ & 4,7 & 67 & $\begin{array}{l}10 \\
0\end{array}$ & 4,6 & $\begin{array}{l}0,6 \\
6\end{array}$ & $\begin{array}{l}5 \\
0\end{array}$ & $\begin{array}{l}4, \\
8\end{array}$ & $\begin{array}{c}0,4 \\
2\end{array}$ & $\begin{array}{l}21 \\
7\end{array}$ & $\begin{array}{l}4, \\
7\end{array}$ & 0,6 \\
\hline al & $\begin{array}{l}\text { RAC } \\
\text { F } 2\end{array}$ & $\begin{array}{l}1, \\
3\end{array}$ & 2,8 & 67 & $\begin{array}{l}10 \\
0\end{array}$ & $\begin{array}{l}3,2 \\
7\end{array}$ & $\begin{array}{l}1,3 \\
7\end{array}$ & $\begin{array}{l}5 \\
0\end{array}$ & $\begin{array}{l}3, \\
7\end{array}$ & $\begin{array}{c}1,4 \\
3\end{array}$ & $\begin{array}{l}21 \\
7\end{array}$ & $\begin{array}{l}3, \\
2\end{array}$ & 1,4 \\
\hline & $\begin{array}{l}\text { RAC } \\
\text { F } 3\end{array}$ & $\begin{array}{l}1, \\
2\end{array}$ & 3,5 & 67 & $\begin{array}{l}10 \\
0\end{array}$ & $\begin{array}{l}3,7 \\
2\end{array}$ & $\begin{array}{l}1,1 \\
8\end{array}$ & $\begin{array}{l}5 \\
0\end{array}$ & $\begin{array}{c}4, \\
1\end{array}$ & $\begin{array}{c}0,9 \\
3\end{array}$ & $\begin{array}{l}21 \\
7\end{array}$ & $\begin{array}{l}3, \\
7\end{array}$ & $\begin{array}{l}1,1 \\
7\end{array}$ \\
\hline
\end{tabular}

Nota 1: RACF 1(som inicial); RACF 2 (som medial) e RACF 3( som final)

Nota 2: $M=$ média $; D P=$ Desvio Padrão

Para as palavras de deteç̧ão do som inicial (itens 1-5 do RACF), os resultados da ANOVA não mostraram efeito estatisticamente significativo para 0 ano escolar $\left(F_{(2,216)}=1,67, p=0,19\right)$, nem para o nível de habilidade de leitura $\left(F_{(2,216)}=2,42, p=0,19\right)$ ou para a interação entre ambos $\left(F_{(2,216)}=0,96, p=0,42\right)$. Para 0 som final (itens 5-10), os resultados da ANOVA mostraram efeito estatisticamente significativo para ano escolar $\left(F_{(2,216)}=5,40 ; p<\right.$ $0,005)$, nível de habilidade de leitura $\left(F_{(2,216)}=5,19, p=0,006\right)$, mas não para a interação entre ambos $\left(F_{(2,216)}=0,67, p=0,61\right)$. 
Comparações post hoc Student Newman Keusl mostraram que os alunos do grupo inferior e médio não diferiram em relação aos escores do RACF, porém, os escores do grupo inferior foram significativamente mais baixos que os do grupo superior. Não houve diferença entre o grupo médio e superior. Para os anos escolares, nas análises post hoc não se identificou diferença significativa entre os grupos.

Para o som medial (itens 10-15), os resultados da ANOVA mostraram efeito estatisticamente significativo para ano escolar $\left(F_{(2,216)}=5,28\right.$, $p=0,006)$, mas não para o nível de habilidade de leitura $\left(F_{(2,216)}\right.$ $=2,95, p=0,054)$ ou para interação entre ambos $\left(F_{(2,216)}=1,09\right.$, $p=0,05)$. Comparações post hoc Student Newman Keusl mostraram que os alunos de segundo e terceiro ano não diferiram em relação aos escores do RACF, porém, os escores das crianças de segundo ano foram significativamente inferiores aos escores dos alunos do quarto ano. Não houve diferença entre o segundo e terceiro ano nessas tarefas.

Considerando o segundo objetivo que visava verificar a evidência de validade de entre medidas que avaliam construtos similares, os escores do RAFC foram comparados com outras tarefas que exigiam processamento fonológico, quais sejam, dois subtestes do WISC III: memória de dígitos e vocabulário. Recorreu-se a provas de correlações bisseriais de Pearson para explorar os dados obtidos. O escore total no RACF correlacionou-se de forma estatisticamente significativa com a memória auditiva de trabalho - substeste WISC III $(R=0,36 . p<0,001)$. Também a correlação entre o escore total no RACF e o escore obtido no vocabulário mostrou correlações significativas, porém de magnitude fraca $(R=0,20 ; p=0,003)$.

\section{Discussão}

Pesquisas que buscam estabelecer a validade de instrumentos de avaliação têm sido cada vez mais frequentes na literatura, visto que a área de psicometria se expandiu nos últimos anos, graças à demonstração de que o uso de medidas para avaliação dos fenômenos não pode ser aleatório, mas sim baseado em evidências sustentadas por dados empíricos obtidos com estudos metodologicamente adequados (Urbina, 2007; Nunes \& Primi, 2010). Os dados do presente trabalho contribuíram para testar a validade do Roteiro de Avaliação da Consciência Fonológica - RACF, enquanto instrumento de rastreio que deveria ser suficientemente sensível para captar diferenças esperadas no desenvolvimento metalinguístico de crianças (Branum-Martin et al., 2006; Santos, 1996, Suehiro \& Santos, 2011). Para tanto, várias análises estatísticas foram feitas e a discussão seguirá a ordem dos resultados apresentados, iniciando 
pelas comparações entre os anos escolares.

Os resultados mostraram que o RACF discrimina entre os anos escolares. As crianças de quarto ano foram melhores no Roteiro de Avaliação da Consciência Fonológica do que as crianças de segundo ano. As crianças de terceiro ano parecem estar num estágio intermediário, uma vez que seus escores não diferiram estatisticamente nem das crianças de segundo, nem das do quarto ano. Esses resultados indicam que essa habilidade continua a se desenvolver ao longo dos primeiros anos de escolarização. Sugerem, no entanto, haver uma mudança qualitativa entre o terceiro e quarto ano quanto a esse desenvolvimento, quando analisamos a posição dos sons avaliados. De um modo geral esses resultados dão suporte empírico às teorias sobre o desenvolvimento da consciência fonológica e apontam o RAFC como um bom instrumento para avaliar esse construto (Bertelson et al., 1989; Bradley \& Bryant, 1993; Cardoso-Martins \& Frith, 1999; Gombert, 1992; 2003; Pestun et al., 2010).

Em relação à discriminação de bons e maus leitores, os resultados indicaram que os leitores no nível inferior e médio no TDE não diferiram em relação aos escores do RACF, porém os escores das crianças do grupo inferior foram significativamente inferiores aos dos leitores superiores. Indicam também que o RAFC foi sensível para detectar essas dificuldades. Assim como no caso do desenvolvimento da consciência fonológica, os resultados aqui dão suporte às teorias sobre a consciência fonológica, que apontam que crianças com dificuldades na leitura e escrita apresentam déficits no processamento fonológico (Bertelson et al., 1989; Miranda \& Mota, 2011).

O RACF foi construído para testar a habilidade de detectar sons em diferentes posições da palavra, visto que teoricamente é esperado que a identificação em cada uma dessas posições tenha graus diferentes de dificuldades (Spinillo et al., 2010; Suehiro \& Santos, 2011). Verificamos a performance das crianças na detecção dos sons nessas diferentes posições. Não houve diferenças entre os anos escolares para o som inicial. Para o som final o resultado da ANOVA foi significativo, mas análises post hoc mais conservadoras não identificaram diferenças. Para o som medial novamente observase um período intermediário para o terceiro ano, como no caso do escore total do RACF.

Vários estudos têm demonstrado que a consciência fonológica interage com a leitura (Barrera \& Maluf, 2003; Capovilla \& Capovilla, 2000; Gombert, 2003; Guimarães, 2003; Phillips et al., 2008). As crianças nesse estudo tinham pelo menos um ano completo de escolarização formal. As tarefas envolvendo o som final e inicial podem ter sido muito fáceis para eles. O som medial parece ser 0 que melhor discriminaria as crianças nessa faixa etária. Assim, esses 
resultados são consistentes com um modelo de desenvolvimento que propõe que consciência fonológica interage com a escolarização.

Embora os resultados das correlações tenham sido fracas, encontramos também evidência de validade para o RACF quando verificamos a associação entre essa tarefa e outras tarefas que avaliam o desenvolvimento linguístico. No caso especifico do processamento fonológico, o RACF correlacionou-se de forma estatisticamente significativa com os escores no subteste de memória auditiva do WISC III. Na introdução desse trabalho, argumentamos que a memória auditiva é uma medida de processamento fonológico (Wagner \& Torgesen, 1987; Castles \& Coltheart, 2004). Dessa maneira, essas correlações apresentam suporte adicional para a validação do RAFC.

\section{Considerações finais}

Os resultados aqui apresentados mostram que o RACF é um instrumento com validade para avaliar a habilidade metafonológica de crianças de segundo ao quarto ano do ensino fundamental. O fato de ele separar os escores dos alunos da amostra por ano escolar e por nível de habilidade fornece evidências de validade de critério para o RACF, visto que nas duas situações foram usadas variáveis externas para aferir a sua sensibilidade como medida do construto consciência fonológica. O resultado alcançado para esse objetivo possibilita identificar o RACF como um instrumento com qualidades psicométricas adequadas para se avaliar a consciência fonológica de crianças na etapa do ensino fundamental.

Também os índices de correlação positiva dos escores obtidos com os escores do eliminar RACF e subtestes de outras medidas de desenvolvimento linguístico indicam que eles estão medindo construtos similares. No entanto, pode-se considerar este resultado como uma das limitações deste estudo, visto que poderiam ter sido explorados outros aspectos qualitativos das medidas, explorando, por exemplo, o tipo de erro cometido no subteste do WISC III e do TDE para que conclusões mais ricas pudessem ser verificadas de modo a favorecer delineamentos futuros de novas pesquisas.

Se partirmos do pressuposto que a habilidade metalinguística é necessária para a aquisição da escrita, como apontam vários estudos estrangeiros e nacionais (Castles \& Coltheart, 2004, Golbert \& Salles, 2010; Pestun et al., 2010; Spinillo et al., 2010; Suehiro \& Santos, 2011, entre outros), os resultados do presente estudo poderiam ser tratados com o propósito de apresentar evidências de validade de um Roteiro de Avaliação da Consciência Fonológica. Esse roteiro poderá servir como um instrumento de rastreio, a ser utilizado tanto por professores no contexto da sala de aula, quanto por 
profissionais na área clínica para explorar hipóteses diagnósticas de crianças que apresentam algum tipo de dificuldade de aprendizagem. Acreditamos que com base nos resultados encontrados, tanto podem ser planejadas práticas escolares para uso em sala de aula, quanto podem ser propostos programas de intervenção remediativos.

\section{Referências}

Alloway, T. P., Gathercole, S. E. Adams, A. M., Willis, C., Eaglen, E. \& Lamont, E. (2005). Working memory and phonological awareness as predictors of progress towards early learning goals at school entry. British Journal of Developmental Psychology, 23, 417-426.

Barrera, S., \& Maluf, M. (2003). Consciência metalinguística e alfabetização: um estudo com crianças da primeira série do ensino fundamental. Psicologia: Reflexão e Crítica, 16(3), 491502.

Bertelson, P., Gelder, B. de, Tfouni, L. V., \& Morais, J. (1989). Metaphonological abilities of adult illiterates: New evidence of heterogeneity. European Journal of Cognitive Psychology, 1(3), 239-250.

Bradley, L, \& Bryant, P. (1983). Categorizing sounds and learning to read - a causal connection. Nature, 301, 419-521.

Bradley, L., \& Bryant, P. (1985). Children's reading problems. Oxford: Basil Blackwell.

Branum-Martin, L., Mehta, P. D., Fletcher, J. M., Carlson, C. D., Ortiz, A., Carlo, M., \& Francis, D. J. (2006). Bilingual phonological awareness: Multilevel construct validation among Spanishspeaking kindergarteners in transitional bilingual education classrooms. Journal of Educational Psychology, 98(1), 170-181

Capovilla, A., \& Capovilla, F. (2000). Efeitos do treino de consciência fonológica em crianças com baixo nível sócio-econômico. Psicologia: Reflexão e Crítica, 13(1), 7-24.

Cardoso-Martins, C. (1995). Consciência fonológica e alfabetização. Petrópolis: Vozes.

Cardoso-Martins, C., \& Frith, U. (1999). Consciência fonológica e habilidade de leitura na Síndrome de Down. Psicologia: Reflexão e Crítica, 12(1), 209-224.

Castles, A., \& Coltheart, M. (2004). Is there a causal link form phonological awareness to success in learning to read? Cognition, 91(1), 77-111.

Cuningham, A. E. (1990). Implicit versus explicit instruction in phonemic awareness. Journal of Experimental Child Psychology, $50,426-444$. 
Gindri, G., Keske-Soares, M., \& Mota, H. B. (2007). Memória de trabalho, consciência fonológica e hipótese de escrita. Pró-Fono Revista de Atualização Científica, 19(3), 313-322.

Golbert, C. S., \& Salles, J. F. (2010). Desempenho em leitura/escrita e em cálculos aritméticos em crianças de $2^{a}$ série, Psicologia Escolar e Educacional, 14(2), 203-210.

Gombert J. E. (1992). Metalinguistic Development. Hertfordshire: Harverster Wheatsheaf.

Gombert, J. (2003). Atividades metalingüísticas e aprendizagem da leitura. In M. R. Maluf. Metalinguagem e aquisição da escrita: contribuições da pesquisa para a prática de alfabetização (pp.19-63). São Paulo: Casa do Psicólogo.

Goswuami, U., \& Bryant, P. (1990). Phonological Skills and Learning to Read. London: Lawrence Erlbaun Associates.

Guimarães, S. R. K. (2003). Dificuldades no desenvolvimento da lectoescrita: o papel das habilidades metalinguísticas. Psicologia: Teoria e Pesquisa, 19(1), 33-45.

Maclean, M., Bryant, P., \& Bradley, L. (1987). Rhymes, nursery rhymes, and reading in early childhood. Merrill-Palmer Quarterly, 33, 255-282.

Miranda, L., \& Mota, M. M. P. E. (2011). Estratégias cognitivas de escrita do português do Brasil. Psico-USF, 16 (2), 227-232.

Morais, J., Content, A., Bertelson, P. Cary, L., \& Alegria, J. (1986). Literacy training and speech segmentation. Cognition, 7, 323331.

Mota, M. M. P. E., \& Guimarães, S. B. (2011) Leitura contextual e o processamento metalinguístico: considerações teóricas, Paidéia, 21(49), 279-283.

Nunes, C. H. S. S., \& Primi, R (2010). Aspectos técnicos e conceituais da ficha de avaliação dos testes psicológicos. Em Conselho Federal de Psicologia - CFP (Org.), Avaliação psicológica: diretrizes na regulamentação da profissão (pp. 101-128). Brasília: CFP.

Pestun, M. S. V., Omote, L. C. F., Barreto; D. C. M., \& Matsuo, T. (2010). Estimulação da consciência fonológica na educação infantil: prevenção de dificuldades na escrita. Psicologia Escolar e Educacional, 14(1), 95-104.

Phillips, B. M., Clancy-Manchetti, J., \& Lonigan, C. J. (2008). Successful phonological awareness instruction with preschool children. Topic in Early Childwood Special Education, 28(1), 317.

Santos, A. A. A. (1996). A influência da consciência fonológica na aquisição da leitura e da escrita. In F. F. Sisto, G. C. Oliveira, L. D. T. Fini, M. T. C. C. Souza, \& R. P. Brenelli (Orgs.), Atuação psicopedagógica e aprendizagem escolar (pp. 213-247). Petrópolis: Vozes. 
Souza, E. C., \& Bandini, H. H. M. (2007). Programa de treinamento de consciência fonológica para crianças surdas bilíngues. Paidéia, 17(36), 123-135.

Spinillo, A. Mota, M. M. P. E., \& Correa, J. (2010). Consciência metalinguística e compreensão de leitura: diferentes facetas de uma relação complexa. Educar em Revista, 38, 157-171.

Stein, L. (1994). Teste de Desempenho Escolar. São Paulo, SP: Casa do Psicólogo.

Suehiro, A. C. S., \& Santos, A. A. A. (2011). Roteiro de Avaliação da Consciência Fonológica (RACF). Acta Colombiana de Psicología, 14(1), 147-154.

Urbina, S. (2007). Fundamentos para a testagem psicológica. Porto Alegre: Artmed.

Wagner, R.K. \& Torgeson, J. K. (1987). The nature of phonological awareness and its causal role in the acquisition of reading skills. Psychological Bulletin, 101, 192- 212.

Wechsler, D. (1991). WISC-III: Escala de inteligência Weschsler para crianças. São Paulo: Casa do Psicólogo.

\section{Endereço para correspondência \\ Márcia Maria Peruzzi Elia da Mota}

Universidade do Estado do Rio de Janeiro

Programa de Pós-Graduação em Psicologia Social

Rua São Francisco Xavier, 524, $10^{\circ}$ andar sala 10019 bloco B, Maracanã, CEP 20550-013, Rio de Janeiro, RJ, Brasil

Endereço eletrônico: mmotapsi@gmail.com

\section{Acácia Aparecida Angeli dos Santos}

Universidade São Francisco

Programa de Pós-Graduação Stricto-Sensu em Psicologia

Rua Alexandre Rodrigues Barbosa, 45, Itatiba, CEP 13251-900, São Paulo, SP, Brasil

Endereço eletrônico: acacia.angeli@gmail.com

\section{Silvia Brilhante Guimarães}

Universidade do Estado do Rio de Janeiro

Programa de Pós-Graduação em Psicologia Social

Rua São Francisco Xavier, 524, $10^{\circ}$ andar sala 10019 bloco B, Maracanã, CEP 20550-013, Rio de Janeiro, RJ, Brasil

Endereço eletrônico: silvia_brilhante@yahoo.com.br

\section{Carolina Conti}

Universidade Federal de Juiz de Fora

Instituto de Ciências Humanas, Departamento de Psicologia

Rua José Lourenço Kelmer, s/n, São Pedro, CEP 36036-900, Juiz de Fora, MG,

Brasil

Endereço eletrônico: carolfconti@yahoo.com

Recebido em: 11/06/2014

Reformulado em: 08/10/2014

Aceito para publicação: 09/10/2014

\section{Notas}


* Doutora em Psicologia pela Universidade de Oxford, docente do Programa de Pós Graduação da Universidade Salgado de Oliveira e do Programa de Pós Graduação em Psicologia Social da UERJ. Bolsista produtividade do CNPq.

** Psicóloga. Doutora em Psicologia Escolar e do Desenvolvimento pela USP. Docente da graduação e do Programa de Pós-graduação Stricto Sensu em Psicologia da Universidade São Francisco. Bolsista Produtividade do CNPq.

*** Mestre em psicologia pela Universidade Federal de Juiz de Fora, Universidade Federal de Juiz de Fora. Doutoranda em Psicologia pela Universidade Estadual do Rio de Janeiro.

**** Psicóloga. Mestre em Psicologia pela Universidade Federal de Juiz de Fora. 